\title{
Investigating Exploration Techniques for ACS in Discretized Real-Valued Environments
}

\author{
Norbert Kozlowski \\ Wroclaw University of Science and Technology \\ Department of Computer Engineering \\ Wroclaw, Poland \\ norbert.kozlowski@pwr.edu.pl
}

\author{
Olgierd Unold \\ Wroclaw University of Science and Technology \\ Department of Computer Engineering \\ Wroclaw, Poland \\ olgierd.unold@pwr.edu.pl
}

\begin{abstract}
One way of dealing with the real-valued input signal is to discretize it. This might influence the process of learning the environmental model by the ACS2 agent. A more sophisticated method of selecting action can be applied to increase the speed of gaining knowledge by determining the most valuable regions of the input-space. This paper compares four ACS2 biasing exploration techniques applied across four real-valued environments. A new class of benchmark problem (inverted pendulum) and an agent modification - Optimistic Initial Quality (OIQ) are introduced for ACS2 both with promising outcomes.
\end{abstract}

\section{CCS CONCEPTS}

- Computing methodologies $\rightarrow$ Rule learning; • Software and its engineering;

\section{KEYWORDS}

Anticipatory Learning Classifier Systems, OpenAI Gym

\section{ACM Reference Format:}

Norbert Kozlowski and Olgierd Unold. 2020. Investigating Exploration Techniques for ACS in Discretized Real-Valued Environments. In Genetic and Evolutionary Computation Conference Companion (GECCO '20 Companion), fuly 8-12, 2020, Cancún, Mexico. ACM, New York, NY, USA, 9 pages. https://doi.org/10.1145/3377929.3398079

\section{INTRODUCTION}

In order to fully meet the capabilities of an Anticipatory Classifier System (ACS) of evolving a complete, accurate and maximally general environmental representation a more advanced exploration strategy might be useful for improving the model and behavioural learning. It can be especially crucial for environments with the input-space size eliminating the possibility of exhaustive search. In the usual trade-off between exploration and exploitation, the exploration usually inhibits the proper usage of current knowledge for optimal behaviour. The methods validated herein focuses mainly on model learning optimization rather than policy learning optimization.

Permission to make digital or hard copies of part or all of this work for personal or classroom use is granted without fee provided that copies are not made or distributed for profit or commercial advantage and that copies bear this notice and the full citation on the first page. Copyrights for third-party components of this work must be honored.

For all other uses, contact the owner/author(s).

GECCO '20 Companion, July 8-12, 2020, Cancún, Mexico

(C) 2020 Copyright held by the owner/author(s).

ACM ISBN 978-1-4503-7127-8/20/07.

https://doi.org/10.1145/3377929.3398079
This work intends to examine the capabilities and performance of ACS2 system on larger input-space settings than used in traditional examples (binary multiplexer or grid environments). Real-valued environments provide different complexity levels and are scalable. The assumption is that by using more sophisticated exploration strategy, the agent will be able to accomplish this task more effectively. Therefore, one new method is proposed and contrasted with existing ones.

This paper is structured as follows. Section 2 describes the Learning Classifier System in general and its anticipatory extension. In Section 3, existing methods of biasing exploration in Learning Classifier Systems are presented, also proposing a new technique based on bandit algorithms. Section 4 introduces four real-valued environments used for estimating the performance and strengths of each approach. A new environment used in control problems - Cart Pole balancing is used for the first time in this class of algorithms. Finally, Section 5 presents the results of experiments, and the conclusion is included in Section 6.

\section{LEARNING CLASSIFIER SYSTEMS (LCS)}

John Holland developed the LCS concept in the 1970s[14]. The term has been used to describe the family of machine learning (ML) algorithms that emerged from a founding concept designed to model complex adaptive systems[29].

The system stores the knowledge in an evolving population $[P]$ of classifiers. Each classifier consists of "IF-THEN" rule describing the environmental state where it can be applied and some additional metrics describing its relevance. New classifiers might be introduced into the population by two main processes - covering and genetic algorithms.

The covering creates new classifiers with a rule matching exactly the environmental state. Then some generalization is introduced randomly. On the other hand, the genetic algorithms (GA) try to take advantage of mixing rules of promising individuals from the population $[P]$.

During the interaction with the environment, all rules are reevaluated continuously changing their metrics accordingly. Classifiers that are correctly describing the consequences of an action are kept in population, while others are discarded.

The most significant benefit of the system is the ability to create human-understandable rules. Also, classifiers by design can map only certain portions of the input space, which allows breaking a more substantial problem into smaller parts.

There are many extensions to basic LCS algorithms, solving various use-cases. One of the approaches is to predict the consecutive 
state after executing particular action, which is fulfilled by the Anticipatory Learning Classifier Systems (ALCS).

\subsection{Anticipatory Learning Classifier Systems (ACS2)}

In 1993 Hoffmann proposed a theory of Anticipatory Behavioral Control [12] that was further refined in [13]. It distinguishes between the following points (visualized in Figure 1):

(1) Any behavioral act or response $(R)$ is accompanied by anticipation of its effects.

(2) The anticipations of the effects $E_{\text {ant }}$ are compared with the real effects $E_{\text {real }}$.

(3) The bond between response and anticipation is strengthened when the anticipations were correct and weakened otherwise.

(4) The $R$ - $E_{\text {ant }}$ relations are further differentiated by behavioral relevant stimuli.

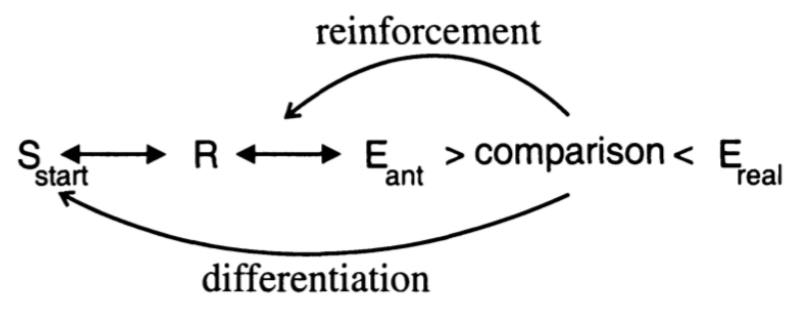

Figure 1: The theory of anticipatory behavioral control. Figure adapted from $[7$, p. 4].

That insight of presence and importance of anticipations in animals and man lead to the conclusion that it would be beneficial to represent and utilize them also in animats.

The first approach was undertaken by Stolzmann in 1997 [20]. He presented a system called ACS ("Anticipatory Classifier System") enhancing the classifier structure with an anticipatory or effect part that anticipates the effects of an action in a given situation. To introduce new classifiers a dedicated component realizing Hoffmann's theory was introduced - Anticipatory Learning Process (ALP).

The ACS starts with a population $[P]$ of most general classifiers ('\#' in a condition and effect parts) for each available action. In order to ensure the presence of a classifier in every consecutive situation it's unable to delete those classifiers. During each behavioral act current perception of environment $\sigma(t)$ is captured. Then, a match set $[M](t)$ is formed, consisting all classifiers from $[P]$ where the condition matches the perception $\sigma(t)$. Next, one classifier $c l$ is drawn from $[M](t)$ using some exploration policy. Usually, an epsilon-greedy technique (described in section 3.1) or roulettewheel selection is used. Then the classifiers action $c l . a$ is executed in the environment, and new perception $\sigma(t+1)$ and reward $\rho(t+1)$ values are presented to the agent. Knowing the classifiers anticipation and current state the ALP module can adjust the condition and effect parts of the classifier $\mathrm{cl}$. Based on this comparison certain cases might occur. The useless case is when no change in perception is perceived from the environment after taking a given action. If so the quality $c l . q$ is decreased. The unexpected case is when the new state $\sigma(t+1)$ does not match the prediction of $c l$.E. A new classifier with matching effect part is then generated. The incorrect one is penalized as before. The final case is the expected case when the new state matches the classifier prediction, increasing it's quality. After the ALP application the RL also updates attributes related to obtained reward $\rho(t+1)$ based on the correctness of the overall prediction.

Later in 2002 Butz presented an extension called ACS2 [7]. Most importantly, he modified the original approach by:

(1) explicit representation of anticipations,

(2) applying learning components across the whole action set $[A]$ (all classifiers from $[M]$ advocating selected action),

(3) introduction of Genetic Generalization module for generating new classifier using promising offsprings

The complete behavioral act is presented on Figure 2 and the algorithm is described thoroughly in [9] [21].

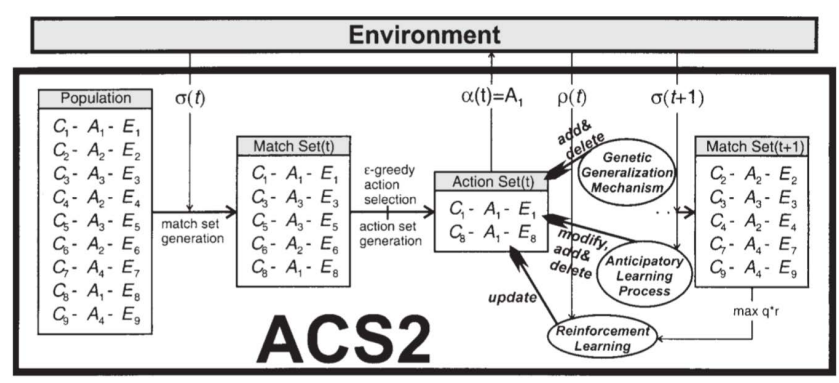

Figure 2: A behavioral act in ACS2 with reinforcement learning (RL) and anticipatory learning process application. Figure adapted from [7, p. 27].

\subsection{Other Anticipatory Classifier Systems}

They were also other approaches in designing anticipatory classifier systems. In 2002 Gerard proposed a YACS (Yet Another Classifier System) [11] that uses heuristics for the latent learning process. In 2005 he also created MACS (Modular Anticipatory Classifier System) [10], where a new formalism makes it possible to use other regularities for the latent learning process generalization. It does not represent situations as an inseparable whole but decorrelates the attributes making it possible to represent regularities across them. Also, in 2005, O'Hara and Bull proposed a combination of XCS with artificial neural networks - X-NCS [19], where a fully connected multilayer perceptron replaced a conventional condition-action rule. Also in 2005 Zatuchna introduced the AgentP [32], created especially for dealing with aliased environments.

The YACS, MACS, X-NCS, AgentP systems are not discussed herein. If the reader is interested in more details, he is invited to consult the cited publications. 


\subsection{Real-Valued Anticipatory Classifier Systems (rACS)}

Unold and Mianowski took the first approach for evaluating the ACS2 system in real-valued environments in 2016 [27]. They obtained promising results on two environments (Corridor and Grid) by discretizing the input-signal.

Later in 2019, Kozlowski and Unold [16] tried to modify the underlying ACS2 processes to utilize the interval-based representation [24]. Satisfactory results were obtained for simple problems, but the ALP mechanism was not able to handle this variant of the alphabet and would require significant changes for effective performance.

This paper uses a standard non-modified version of ACS2. The only difference to Butz [9] version was performed on the detector layer and the extended configuration. For the input signal, the detector has advanced possibilities to discretize it granularly (each attribute separately). The researcher can specify the input range and the desired number of bins. This allows a customizable way of changing the perception accuracy of the system. Additionally, the agent configuration was extended, allowing it to specify the initial quality parameter $q_{0}$, outlined in Section 3. The flow of processes like Anticipatory Learning Process, Genetic Algorithms, and Reinforcement Learning were kept intact.

\section{BIASED EXPLORATION}

At first, model learning capabilities in ALCS was enhanced by Stolzmann and Butz by using behavioral capabilities (internal reinforcement learning or lookahead action selection) in [23] and by introducing the action planning mechanism [22,28].

Later, Butz suggested that by using computationally inexpensive methods by biasing exploration towards specially chosen regions of search-space, the model can be learned locally [6].

This section presents four approaches to deciding which action to choose. First, a baseline method - epsilon-greedy, which is a default option for LCS, will be described. Later two methods introduced by Butz - action-delay and knowledge-array bias. They examine the match set $[M]$ searching for indications which action might result in the highest information gain. Eventually, the latter approach is inspired by an "Optimistic Initial Values" approach described by Sutton in [25, p.34]. This strategy turned out to be very effective for Multi-armed bandit problems, where the main objective is to select the most promising action and was never examined in any LCS domain.

\subsection{Epsilon-Greedy (EG)}

In the epsilon-greedy approach, the agent discovers all regions from the input-space equally, not favoring any specific behavior. In each step, random action is executed with $p_{\text {explr }}$ probability. Then it is chosen uniform randomly from classifiers composing a match set $[M]$. In the case of $1-p_{\text {explr }}$, action from the most fitted classifier is executed. By doing so, the agent can occasionally perform the move he thinks is the best at a given time, reinforcing its beliefs about the consequences.

\subsection{Action-Delay (AD)}

This bias is based on the recency-based principle assuming that the action executed a long time ago, might introduce new situationaction-effect triples. To determine which action was executed most long ago at the current time $t$ the $t_{\text {alp }}$ field of all classifiers in match set $[M](t)$ is analyzed. For situation $\sigma(t)$ the action of classifier $c l$ with the lowest value of $t_{a l p}$ is selected.

In case if there exists an action not represented by any classifier in $[M](t)$ that it is assumed to be experienced most long ago (if ever) and therefore chosen for execution.

\subsection{Knowledge Array (KA)}

This method, on the contrary, is based on the error-based principle. For ACS2, a quality $q$ denoting the accuracy of predictions for each classifier can be used to measure it.

The bias generates the knowledge array KA from classifiers in a match set $[M]$ in which each entry specifies the averaged quality for the anticipation for each possible action - see Equation 1.

$$
K A[a]=\frac{\sum_{c l \in[M] \wedge c l . A=a} c l . q \cdot \text { cl.num }}{\sum_{c l \in[M] \wedge c l . A=a} \text { cl.num }}
$$

An action with the lowest value in the knowledge array is selected for execution. Similarly, as in the action delay bias, if there are actions not expressed by any classifiers, they are chosen first.

\subsection{Optimistic Initial Quality (OIQ)}

By default, newly created classifiers $c l$ have the initial quality set to $c l . q=0.5$. It can be said that they are biased towards their initial quality. In practice, it should not be a problem because this value will converge to optimal ones over a set of trials. Changing this parameter provides an easy way of supplying the agent with the confidence of the generated classifiers.

In this method, the behavior of the agent was parametrized by an extra parameter - initial quality - $q_{0}$. Every time a new classifier is generated, its quality is set to new value $c l . q=q_{0}$.

In all the experiments, there was fixed $q_{0}=0.8$, with the expectation that the agent will be able to build an internal model of knowledge representation faster (especially in early stages). For the action selection strategy, the default epsilon-greedy method was chosen.

\section{REAL-VALUED ENVIRONMENTS}

All environments were created accordingly to the OpenAI Gym interface and are available publicly ${ }^{1}$.

\subsection{Corridor}

The corridor is a 1D multi-step, linear environment introduced by Lanzi to evaluate the XCSF agent [18]. The system output is defined over a finite discrete interval $[0, n]$. On each trial, the agent is placed randomly on the path and can execute two possible actions - move left or right (which corresponds to moving one unit in a particular direction - see Figure 3). The trial ends when it reaches the final state $n$ (obtaining reward $r=1000$ ) or when the maximum number of steps is exceeded.

\footnotetext{
${ }^{1}$ https://github.com/ParrotPrediction/openai-envs
} 


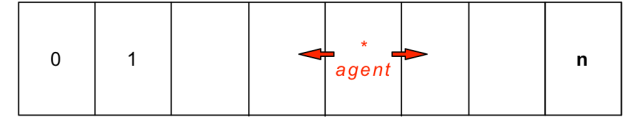

Figure 3: Corridor environment. The size of the input space is $n$.

In [18], Lanzi used a real-valued version if this environment where the agent location was described by a value between $[0,1]$. When it executed an action, a predefined step-size was added to the current position, changing its value. When the agent reaches the final state $s=1.0$, the reward is paid out.

The environment examined herein represents the state already in discretized form.

\subsection{Grid}

Grid is an extension of the Corridor environment [18]. A vertical dimension and two new actions (move up, move down) are added. The state is now identified as a pair of integers, reflecting the agent's position on board. Similarly, the goal is to reach the reward located at position $(n, n)$ - see Figure 4 for reference.

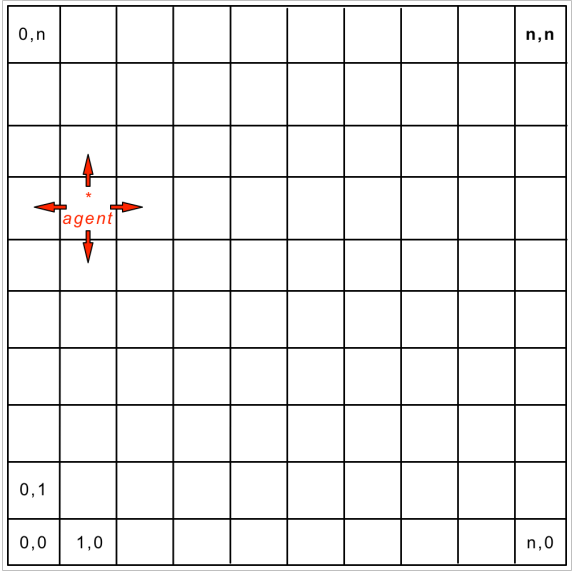

Figure 4: Grid environment. The input space size is $n^{2}$.

\subsection{Real Multiplexer (rMPX)}

The Real Multiplexer is the modification to the traditional Boolean Multiplexer (MPX) introduced by Wilson [30] to examine the performance in single-step environments using real-valued data.

The standard version is, however, still not suitable to be used with ACS2. Because in order to form new classifiers, an agent utilizes the fact of perceptual causality, assuming that after executing an action, the state will change. The MPX does not have any possibility to send any feedback about the correctness of the action.

Butz suggested two solutions to this problem [7, p. 55]. The following paper assumes that the state generated by rMPX is extended by one extra bit, denoting whether the classification was successful. A detailed example can be found in [16].
Also, the discretizer is used, converting real-valued numbers into integers. By this fact, another parameter is introduced - a number of bins $k$. Its value is directly responsible for controlling the accuracy of generated classifiers - for example, see Table 1.

Thus, the input space of the environment can be calculated as $2 k^{n}$, where $k$ is the number of bins and $n$ the length of the MPX signal.

\begin{tabular}{|l|l|l|l|l|l|l|l|}
\hline Original & 0.86 & 0.29 & 0.56 & 0.89 & 0.33 & 0.49 & 0.00 \\
\hline Discretized & 5 & 1 & 3 & 5 & 2 & 2 & 0 \\
\hline
\end{tabular}

Table 1: Example of discretizing real-valued input using 6 bins. A random perception of 6-bit rMPX extended with 1 bit (last column) was used. Size of the input space is $2 \cdot 6^{6}=93312$.

\subsection{Cart Pole}

Barto introduced the Cart Pole environment as a reinforcement learning control problem [4]. The task is to balance a pole that is hinged to a movable cart by applying forces (move left or move right) to the cart's base (Figure 5). The system starts upright, and its goal is to prevent the stick from falling over. After each step, a reward of +1 is provided. The episode ends when the pole is more than 15 degrees from vertical, or the cart moves more than 2.4 units from the center ${ }^{2}$. Table 2 presents the agent's perception of the environment.

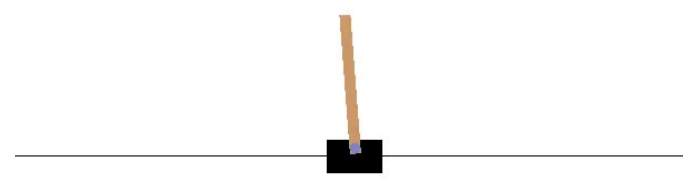

Figure 5: CartPole render from the OpenAI Gym.

\begin{tabular}{|l|l|l|l|}
\hline Num & Observation & Min & Max \\
\hline$\sigma_{0}$ & Cart Position & -2.4 & 2.4 \\
\hline$\sigma_{1}$ & Cart Velocity & $-\infty$ & $\infty$ \\
\hline$\sigma_{2}$ & Pole Angle & $\sim-41.8^{\circ}$ & $\sim 41.8^{\circ}$ \\
\hline$\sigma_{3}$ & Pole Velocity at Tip & $-\infty$ & $\infty$ \\
\hline
\end{tabular}

Table 2: Agent's observation of the CartPole environment.

The environment is considered as solved if the average reward if greater than or equal to 195 over last 100 trials.

${ }^{2}$ https://gym.openai.com/envs/CartPole-v0 


\section{EXPERIMENTS}

All action selection mechanisms were tested throughout four different benchmark problems - Corridor, Grid, Real-Multiplexer, and CartPole. Each subsection presents parameters used for ACS2 configuration, evaluation criteria, and actual experiments setup.

The subsequent research was done in Python 3.7 language using an open-source library PyALCS ${ }^{3}$ [15] and OpenAI Gym [5] and is fully reproducible online (MyBinder.org, interactive Jupyter notebooks) ${ }^{4}$.

\subsection{Corridor}

Three metrics - knowledge, the number of steps in each trial, and overall population count were selected to evaluate the performance of the environment.

Knowledge represents the ability of latent-learning. It is calculated as the presence of reliable classifiers capable of predicting all possible transitions from the given state in the environment (for example, for state 6 , reliable classifiers are present and define how to get to states 5 and 7).

The number of steps is used to verify the exploitation (reinforcement learning) capabilities of the agent after the performing exploration phase.

Due to the performance issues, in all cases, the corridor size was set to $n=20$, but the overall metrics look similar for larger sizes (tested also with values of $n=40$ and $n=100$ ). Additionally, the cross-over capability of the agent was switched off because of the unit length of the perception vector $\sigma$.

Fig 6 presents the results. All methods converge to optimal population size, and after switching to the exploitation mode are able to utilize gained knowledge fully. Regardless of the exploration technique chosen, the agent can obtain full knowledge of the environment in about 20 trials. All advanced techniques seem to reach this point faster than the baseline (EG), with the OIQ method being the fastest one.

The $\mathrm{AD}$ and KA methods accelerate the process of investigating the search-space resulting in earlier classifier creation.

Finally, the agent can fully exploit the environment after switching to "exploit" mode performing a minimal number of steps to reach the goal in each trial. Its also worth mentioning the constant effect of the KA method in the explore phase, not taking the optimal actions most of the time, by continually updating the assumptions about all possibilities.

\subsection{Grid}

Performance plot using Grid of size $n=20$ in Figure 7 shows that regardless of exploration technique chosen, the agent is still able to obtain full knowledge of the environment (faster than in Corridor) and converge with the number of optimal classifiers (KA method here also creates much more classifiers at the beginning of the experimentation).

What is interesting is that the agent is unable to exploit the environment even though he knows the exact consequences of each action (non-optimal number of steps in the exploitation phase). After investigation, it was found out that that most of the classifiers

\footnotetext{
${ }^{3}$ https://github.com/ParrotPrediction/pyalcs

${ }^{4}$ https://github.com/ParrotPrediction/pyalcs-experiments
}

have very similar $r$ value, which represents expected future reward. The reason for this was already spotted for XCS. The cause is the presence of long action chain required to reach the reward causing uniformly spaced reward levels.

The problem was spotted by Lanzi when using XCS in long action chain environments [17]. The cause was the inequality in the exploration of all states in the environment or in the long action chain, which allows over-general classifiers to appear accurate. To solve this, he used some exploration policies (biased exploration and teletransportation) and operators (Specify).

Then, Barry noted that XCS was having problems while exploiting even simple environments with moderate payoff delays [1, 3]. He concludes that XCS is unable to learn proper generalizations for early states in the long action chains. It is partially due to the discount and the reduction in payoff prediction values in classifiers covering these early states, which makes the prediction values sufficiently similar. To solve this problem, he modified the error computation in XCS, which calculates the classifiers' error parameter relative to the magnitude of the payoff to each classifier [2]. His preliminary studies showed promising results.

Later, Butz et al. examined differences between plain tabular Q-learning and Q-learning with gradient descent within XCS [8]. Experiments showed that the gradient descent version makes the algorithm more robust and efficient when solving large and challenging problems.

Nevertheless, every examined approach employed by XCS was still discounting prediction values in every step, making it similar after enough steps. Tharakannel and Goldberg took a different approach by maximizing the average of successive rewards instead of discounted rewards [26]. Their system AXCS performed the same as the original, unmodified XCS on one multi-step environment.

Zang proposed the replacement of Q-learning with R-learning [31]. His modification results in a system that is rapid and able to solve large maze problems. Besides, it produces uniformly spaced payoff levels, which can support long action chains and thus effectively prevent the occurrence of over-generalization.

Hence, the ACS2 algorithm requires additional research in terms of modifying Q-learning reinforcement learning for this class of problems. Three grid size were examined (20, 40 and 100) and the environment was unsolved for all the cases.

\subsection{Real-MPX}

The rMPX is the only single-step environment examined. The goal was to see if the increased search-space will also allow for the optimal performance and population size convergence. In the experiment, the exploration and exploitation phases were executed alternately. The cross-over capabilities were disabled because of the last bit in perception $\sigma$ was denoting the prediction result.

Figure 8 shows that the selection of the exploration technique does not influence the average reward collected over time in all cases. The exploration steps (faded colors) oscillate around 500, which corresponds to random guessing for the correct answer. For the number of reliable classifiers describing the environment, the KA method creates slightly more rules than other methods. 


\section{Performance of [corridor-20-v0] environment}
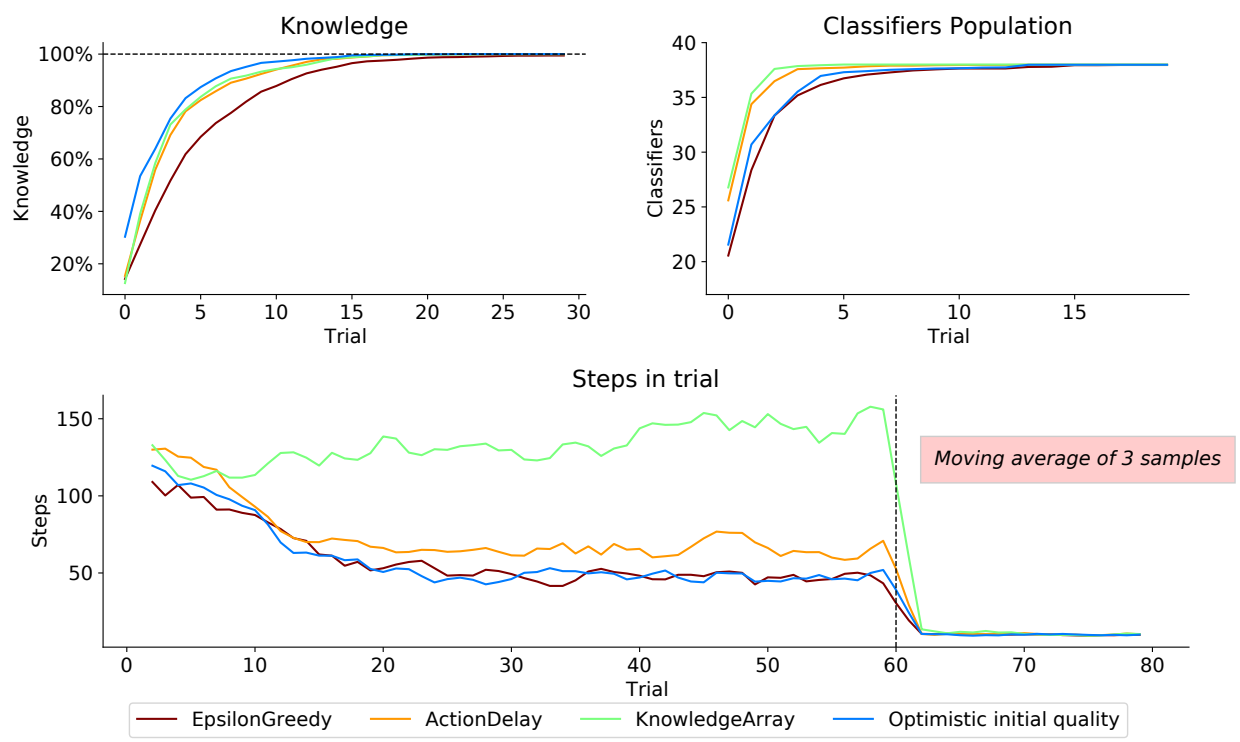

Figure 6: Performance of Corridor environment. ACS2 parameters: $\beta=0.2, \gamma=0.95, \theta_{r}=0.9, \theta_{i}=0.1, \epsilon=0.8 \theta_{G A}=50, \theta_{A S}=20$, $\theta_{\text {exp }}=50, m_{u}=0.03, \chi=0.60$ exploration and 20 exploitation trials averaged over 50 runs. Steps in a trial was plotted with moving-average of 3 last steps for clarity. No explicit discretizer was needed. Maximum number of steps in a trial is 200 . Dotted vertical line indicates the execution of explore and exploit phases.
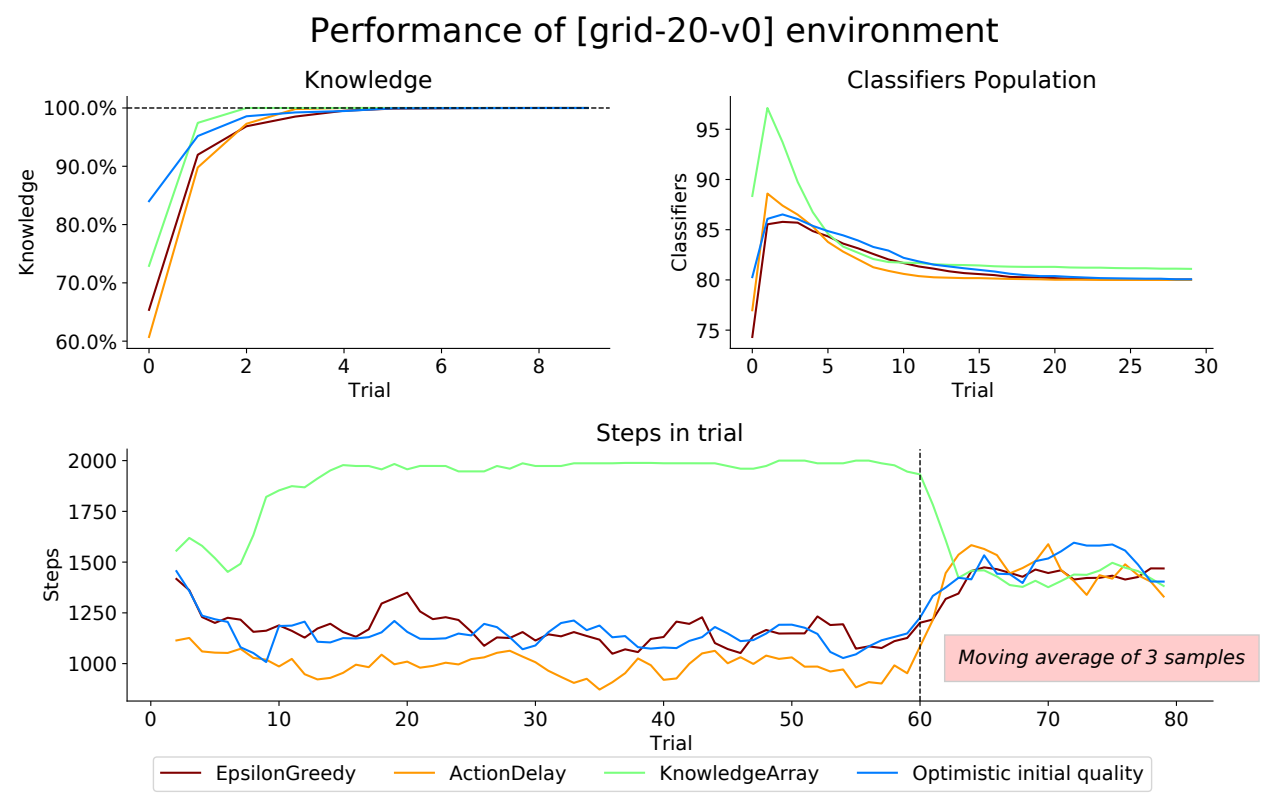

Figure 7: Performance of Grid environment. ACS2 parameters: $\beta=0.2, \gamma=0.95, \theta_{r}=0.9, \theta_{i}=0.1, \epsilon=0.8 \theta_{G A}=50, \theta_{A S}=20$, $\theta_{\text {exp }}=50, m_{u}=0.03, u_{\max }=1, \chi=0.8 .60$ exploration and 20 exploitation trials averaged over 50 runs. Steps in a trial was plotted with moving-average of 3 last steps for clarity. No explicit discretizer was needed. Maximum number of steps in a trial is 2000. Dotted vertical line indicates the execution of explore and exploit phases.

This experiment showed that all exploration techniques are almost equally suitable for the environment possessing the properties of epistasis and heterogeneity. 


\section{Performance of [real-multiplexer-6bit-v0] environment discretized with 6 bins}
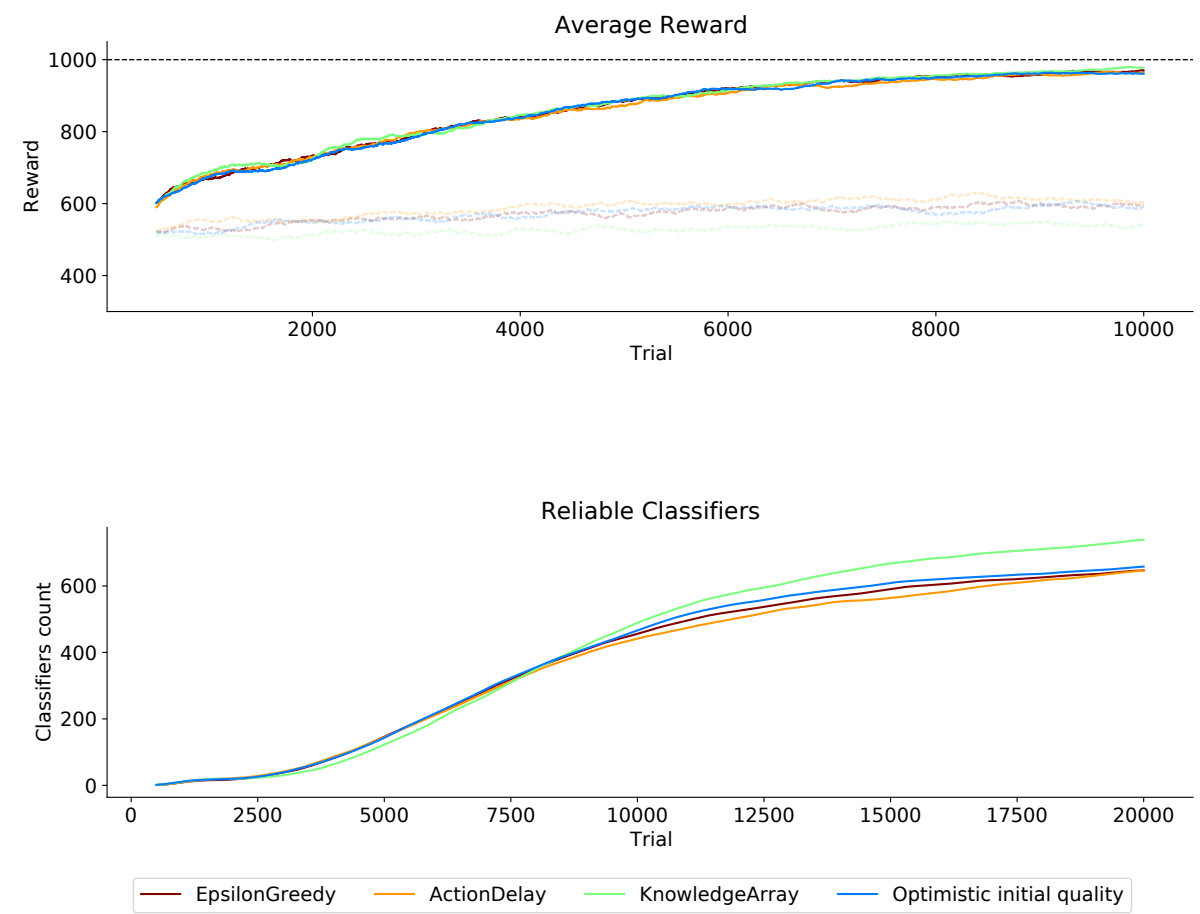

Figure 8: Performance of Real Multiplexer environment. ACS2 parameters: $\beta=0.2, \gamma=0.95, \theta_{r}=0.9, \theta_{i}=0.1, \epsilon=0.8 \theta_{G A}=50$, $\theta_{A S}=20, \theta_{\exp }=50, m_{u}=0.03, u_{\max }=4, \chi=0.0 .2000$ trials were executed in alternating explore-exploit mode averaged over 10 runs. Faded lines shows performance of explore mode, solid ones for exploit.

\subsection{Cart Pole}

The CartPole environment was the most complicated one due to several issues. First, some perception attributes (like cart and pole velocity) are not bounded in any specified range $\sigma_{i} \in[-\infty, \infty]$. To estimate it, data was collected manual, by performing repetitious experiments. Similarly, the extension of the rMPX discretizer was used. In this case, it is possible to specify the number of buckets for each feature independently, varying the input-space size.

In Figure 9, the environment was tested by setting the cart velocity range to $\sigma_{1} \in[-0.5,0.5]$ and pole velocity to $\sigma_{3} \in[-3500,3500]$. The discretizer was experimentally set to only take into consideration $\sigma_{2}$ and $\sigma_{3}$, dividing them into six buckets. Because each perception can be discretized differently, the cross-over was turned-off $\chi=0.0$.

As a result, the agent can keep the pole upright for about 175 steps in each trial after performing just 500 learning trials. This score was possible for every method except the $\mathrm{AD}$. On the other side, $\mathrm{AD}$ was able to create more reliable classifiers quicker than other methods. The performance of the experiment turned out to be very sensitive to the discretization bins chosen.

In official terms, the environment is still not solved. However, it turned out that the number of reliable classifiers required to obtain such a score is less than 10 . That allows a very compact and human-readable form of storing knowledge (see Table 3 for example). It can be seen that the majority of reliable classifiers are marked on the first two attributes, meaning that they should be more distinguishable (for example by increasing discretization).

\begin{tabular}{|c|c|c|c|}
\hline Rule & Mark & Quality & Reward \\
\hline$\# \# 32-1-\# \# \# \#$ & $\{0\},\{0\}, \varnothing, \varnothing$ & 0.95 & 3.32 \\
\hline$\# \# 23-0-\# \# \# \#$ & $\{0\},\{0\}, \varnothing, \varnothing$ & 0.92 & 3.33 \\
\hline$\# \# 23-1-\# \# 2$ & $\{0\},\{0\}, \varnothing, \varnothing$ & 0.90 & 3.04 \\
\hline$\# \# 22-1-\# \# \# \#$ & $\{0\},\{0\}, \varnothing, \varnothing$ & 0.96 & 2.78 \\
\hline$\# \# 33-0-\# \# \# \#$ & $\{0\},\{0\}, \varnothing, \varnothing$ & 0.99 & 2.68 \\
\hline$\# \# 43-1-\# \# \# \#$ & $\{0\},\{0\}, \varnothing, \varnothing$ & 0.95 & 1.47 \\
\hline$\# \# 12-0-\# \# \# \#$ & $\{0\},\{0\}, \varnothing, \varnothing$ & 0.97 & 1.44 \\
\hline$\# \# 12-1-\# \# \# \#$ & $\varnothing, \varnothing, \varnothing, \varnothing$ & 0.97 & 1.38 \\
\hline$\# \# 43-0-\# \# \#$ & $\varnothing, \varnothing, \varnothing, \varnothing$ & 1.00 & 1.34 \\
\hline
\end{tabular}

Table 3: Example of all reliable classifiers obtained after 500 exploration trials in CartPole environment sorted by fitness value.

The experimentation is very sensitive to changes in boundaries and discretizer values. Performing more exhaustive parameter optimization might succeed in obtaining better scores. 


\section{Performance of [CartPole-v0] environment discretized with $(1,1,6,6)$ buckets}
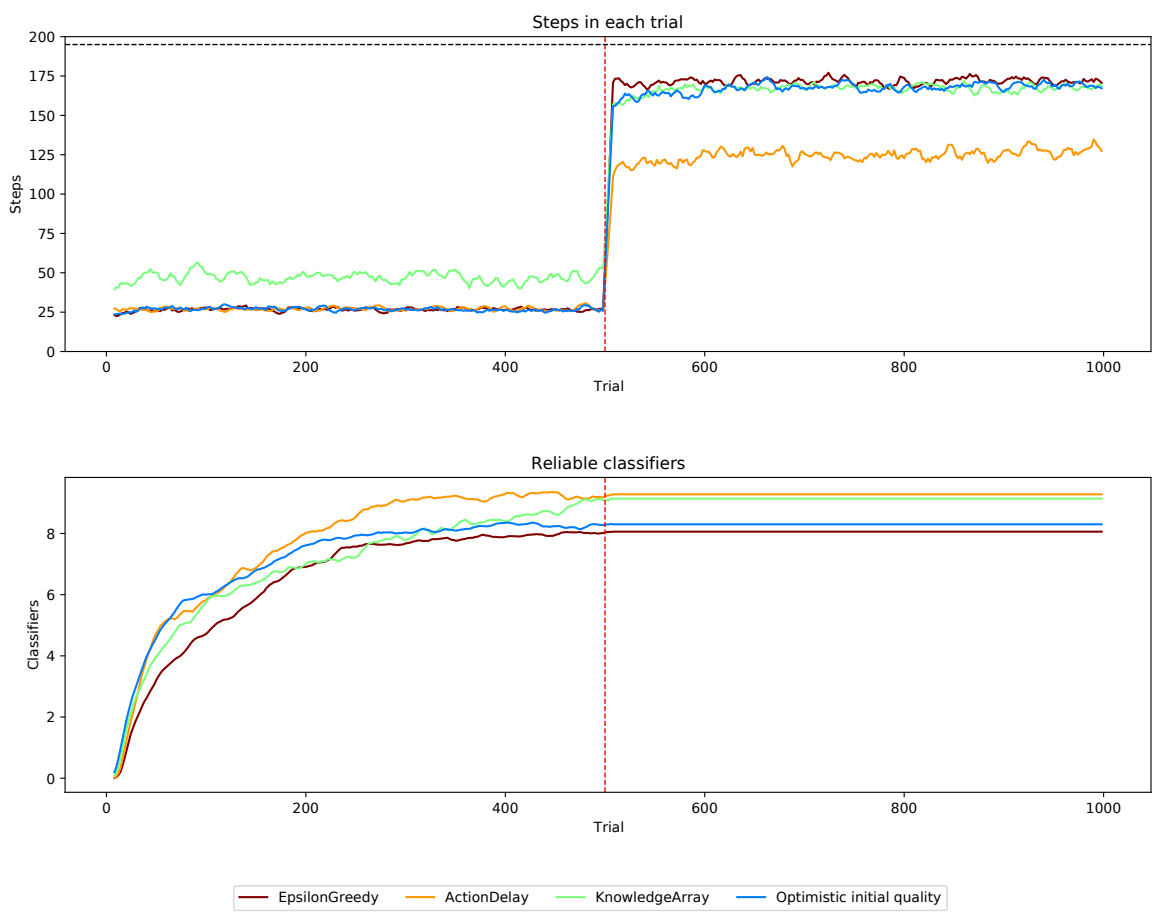

Figure 9: Performance of CartPole environment. ACS2 parameters: $\beta=0.01, \gamma=0.995, \theta_{r}=0.9, \theta_{i}=0.1, \epsilon=0.9 \theta_{G A}=50, \theta_{A S}=20$, $\theta_{\text {exp }}=50, m_{u}=0.03, u_{\max }=4, \chi=0.0 .500$ explore trials and 500 exploit trials averaged over 50 runs. Moving average of 5 last trials applied for clarity. Dotted vertical line indicates the execution of explore and exploit phases.

\section{CONCLUSIONS}

The paper compares four exploration techniques across four environments possessing real-valued properties when using the ACS2 agent. Relevant metrics were collected, and the condensed results are summarized below.

First, the basic version of ACS2 can deal with real-valued environments with just a simple modification of discretizing an input signal. A control problem of an inverted pendulum (Cart Pole) was never evaluated with any learning classifier system before. ACS2 resulted in promising outcomes, generating less than ten classifiers describing its dynamics (presented in Table 3).

Second, the choice of exploration biasing technique has an impact on model learning optimization, especially in the early phases of learning. It was shown that almost all methods provide an improvement in the rate of generating reliable classifiers over a default epsilon-greedy strategy. For simple environments like Corridor and Grid, where it is possible to evaluate the complete knowledge of the agent, the OIQ method resulted in the fastest convergence of knowledge. It is caused mainly by the fact that the knowledge is calculated from classifiers' quality, which is directly influenced by higher $q_{0}$ value.

Third, applying granular discretization results in a rapid increase in population size. It can be observed mainly in the Real-Multiplexer case, where the trade-off between a number of bins and population size is visible. An investigation for both automatically determining proper discretization value (like meta-heuristics) and post-processing classifiers for readability might be valuable.

Also, a problem with distributing rewards in environments with longer action chain was spotted (Grid environment). A similar problem was already solved for XCS algorithms but never studied for ACS (see Section 5.2). Therefore some investigation here, like adding R-learning modification, would also be valuable.

Similar investigation for other types of Anticipatory Learning Classifier Systems, like YACS, MACS, X-NCS, would also provide insights about the pros/cons of each algorithm when dealing with potentially increased environmental input-space.

Lastly, the open-source PyALCS library was modified accordingly enabling possibility to use the OIQ method and allowing fine-grained discretization for all compatible environments.

\section{REFERENCES}

[1] Alwyn Barry. 2000. XCS Performance and Population Structure in Multi-Step Environments. Ph.D. Dissertation. Queen's University of Belfast.

[2] Alwyn Barry. 2003. Limits in long path learning with XCS. In Genetic and Evolutionary Computation Conference. Springer, 1832-1843.

[3] Alwyn M Barry. 2002. The stability of long action chains in XCS. Soft Computing 6, 3-4 (2002), 183-199.

[4] Andrew G Barto, Richard S Sutton, and Charles W Anderson. 1983. Neuronlike adaptive elements that can solve difficult learning control problems. IEEE transactions on systems, man, and cybernetics 5 (1983), 834-846. 
[5] Greg Brockman, Vicki Cheung, Ludwig Pettersson, Jonas Schneider, John Schulman, Jie Tang, and Wojciech Zaremba. 2016. Openai gym. arXiv preprint arXiv:1606.01540 (2016).

[6] Martin V Butz. 2001. Biasing exploration in an anticipatory learning classifier system. In International Workshop on Learning Classifier Systems. Springer, 3-22.

[7] Martin V Butz. 2002. Anticipatory learning classifier systems. Vol. 4. Springer Science \& Business Media.

[8] Martin V Butz, David E Goldberg, and Pier Luca Lanzi. 2005. Gradient descent methods in learning classifier systems: Improving XCS performance in multistep problems. IEEE Transactions on Evolutionary Computation 9, 5 (2005), 452-473.

[9] Martin V. Butz and Wolfgang Stolzmann. 2002. An Algorithmic Description of ACS2. In Advances in Learning Classifier Systems, Pier Luca Lanzi, Wolfgang Stolzmann, and Stewart W. Wilson (Eds.). Springer Berlin Heidelberg, Berlin, Heidelberg, 211-229.

[10] Pierre Gérard, Jean-Arcady Meyer, and Olivier Sigaud. 2005. Combining latent learning with dynamic programming in the modular anticipatory classifier system. European fournal of Operational Research 160, 3 (2005), 614-637.

[11] Pierre Gerard, Wolfgang Stolzmann, and Olivier Sigaud. 2002. YACS: a new learning classifier system using anticipation. Soft Computing 6, 3-4 (2002), 216228.

[12] Joachim Hoffmann. 2016. Vorhersage und erkenntnis. (2016).

[13] Joachim Hoffmann and Albrecht Sebald. 2000. Lernmechanismen zum Erwerb verhaltenssteuernden Wissens. Psychologische Rundschau (2000).

[14] John H Holland and Judith S Reitman. 1978. Cognitive systems based on adaptive algorithms. In Pattern-directed inference systems. Elsevier, 313-329.

[15] Norbert Kozlowski and Olgierd Unold. 2018. Integrating anticipatory classifier systems with OpenAI gym. In Proceedings of the Genetic and Evolutionary Computation Conference Companion. ACM, 1410-1417.

[16] Norbert Kozlowski and Olgierd Unold. 2019. Preliminary tests of a real-valued anticipatory classifier system. In Proceedings of the Genetic and Evolutionary Computation Conference Companion. 1289-1294.

[17] Pier Luca Lanzi et al. 1997. A Study of the Generalization Capabilities of XCS.. In ICGA. Citeseer, 418-425.

[18] Pier Luca Lanzi, Daniele Loiacono, Stewart W Wilson, and David E Goldberg. 2005. XCS with computed prediction in continuous multistep environments. In 2005 IEEE Congress on Evolutionary Computation, Vol. 3. IEEE, 2032-2039.
[19] Toby O'Hara and Larry Bull. 2005. Building anticipations in an accuracy-based learning classifier system by use of an artificial neural network. In 2005 IEEE Congress on Evolutionary Computation, Vol. 3. IEEE, 2046-2052.

[20] Wolfgang Stolzmann. 1997. Antizipative classifier systems. Ph.D. Dissertation. Fachbereich Mathematik/Informatik, University of Osnabrück.

[21] Wolfgang Stolzmann. 2000. An Introduction to Anticipatory Classifier Systems. In Learning Classifier Systems, Pier Luca Lanzi, Wolfgang Stolzmann, and Stewart W. Wilson (Eds.). Springer Berlin Heidelberg, Berlin, Heidelberg, 175-194.

[22] Wolfgang Stolzmann and Martin Butz. 1999. Latent learning and action planning in robots with anticipatory classifier systems. In International Workshop on Learning Classifier Systems. Springer, 301-317.

[23] Wolfgang Stolzmann, Martin V Butz, and David E Goldberg. 2000. First Cognitive Capabilities in the Anticipatory Classifer System. et al.[228] (2000), 287-296.

[24] Christopher Stone and Larry Bull. 2003. For real! XCS with continuous-valued inputs. Evolutionary Computation 11, 3 (2003), 299-336.

[25] Richard S Sutton and Andrew G Barto. 2018. Reinforcement learning: An introduction. MIT press.

[26] Kurian Tharakunnel and David E Goldberg. 2002. XCS with average reward criterion in multi-step environment. (2002).

[27] Olgierd Unold and Marcin Mianowski. 2016. Real-Valued ACS Classifier System: A Preliminary Study. In Proceedings of the 9th International Conference on Computer Recognition Systems CORES 2015. Springer, 203-211.

[28] Olgierd Unold, Edyta Rogula, and Norbert Kozłowski. 2019. Introducing Action Planning to the Anticipatory Classifier System ACS2. In International Conference on Computer Recognition Systems. Springer, 264-275.

[29] Ryan J. Urbanowicz and Will N. Browne. 2017. Introduction to Learning Classifier Systems (1st ed.). Springer Publishing Company, Incorporated.

[30] Stewart W Wilson. 1999. Get real! XCS with continuous-valued inputs. In International Workshop on Learning Classifier Systems. Springer, 209-219.

[31] Zhaoxiang Zang, Dehua Li, Junying Wang, and Dan Xia. 2013. Learning classifier system with average reward reinforcement learning. Knowledge-Based Systems 40 (2013), 58-71.

[32] Zhanna V Zatuchna. 2005. AgentP: a learning classifier system with associative perception in maze environments. Ph.D. Dissertation. University of East Anglia. 